PROCEEDINGS OF THE

AMERICAN MATHEMATICAL SOCIETY

Volume 132, Number 9 , Pages $2511-2515$

S 0002-9939(04)07490-8

Article electronically published on April 8, 2004

\title{
BIRATIONAL MORPHISMS OF THE PLANE
}

\author{
VLADIMIR SHPILRAIN AND JIE-TAI YU
}

(Communicated by Bernd Ulrich)

\begin{abstract}
Let $A^{2}$ be the affine plane over a field $K$ of characteristic 0 . Birational morphisms of $A^{2}$ are mappings $A^{2} \rightarrow A^{2}$ given by polynomial mappings $\varphi$ of the polynomial algebra $K[x, y]$ such that for the quotient fields, one has $K(\varphi(x), \varphi(y))=K(x, y)$. Polynomial automorphisms are obvious examples of such mappings. Another obvious example is the mapping $\tau_{x}$ given by $x \rightarrow x, y \rightarrow x y$. For a while, it was an open question whether every birational morphism is a product of polynomial automorphisms and copies of $\tau_{x}$. This question was answered in the negative by P. Russell (in an informal communication). In this paper, we give a simple combinatorial solution of the same problem. More importantly, our method yields an algorithm for deciding whether a given birational morphism can be factored that way.
\end{abstract}

\section{INTRODUCTION}

Let $K[x, y]$ be the polynomial algebra in two variables over a field $K$ of characteristic 0 , and let $A^{2}$ be the affine plane over $K$. Birational morphisms of $A^{2}$ are mappings $A^{2} \rightarrow A^{2}$ given by polynomial mappings $\varphi$ of the algebra $K[x, y]$ such that for the quotient fields, one has $K(\varphi(x), \varphi(y))=K(x, y)$. Polynomial automorphisms are obvious examples of such mappings. Another obvious example is the mapping $\tau_{x}$ given by $x \rightarrow x, y \rightarrow x y$. Call either of those mappings a simple affine contraction. For a while, it was an open question whether every birational morphism is a product of simple affine contractions. This question was answered in the negative by Russell (in an informal communication). The paper [4] by Daigle contains an elaboration of Russell's methods.

In this paper, we use an altogether different method of "peak reduction" to easily establish the same result. More importantly, our method gives an algorithm for deciding whether a given birational morphism can be factored that way:

Theorem 1.1. Let $K$ be an algebraically closed field. Then there is an algorithm for deciding whether a given birational morphism of $A^{2}$ over $K$ is a product of simple affine contractions.

Here we assume that we are able to perform calculations in the ground field $K$, which basically means that, given (arithmetic expressions for) two elements of $K$, we can decide whether or not they are equal.

Received by the editors November 13, 2002.

2000 Mathematics Subject Classification. Primary 14E07, 14E25; Secondary 14A10, 13B25.

Key words and phrases. Affine plane, birational morphisms, peak reduction.

The second author was partially supported by RGC Grant Project 7126/98P. 
Our method can also be applied to fields that are not algebraically closed to prove that a particular birational morphism is not a product of simple affine contractions.

Proposition 1.2. Let $K$ be any field of characteristic 0 , and let $\phi: x \rightarrow u, y \rightarrow v$ be a birational morphism of $A^{2}$ over $K$. If $\phi$ is a product of simple affine contractions, then the sum of the degrees of $u$ and $v$ can be decreased by a single generalized simple affine contraction.

Generalized simple affine contractions are morphisms of the types (ET1)-(ET3), defined in Section 2.

For example, the following birational morphism of $A^{2}$ over $\mathbf{R}$ is not a product of simple affine contractions (see Example 2 at the end of Section 2): $x \rightarrow x, y \rightarrow$ $y x^{2}+y$. To find a birational morphism of $A^{2}$ over $\mathbf{C}$ that is not a product of simple affine contractions is somewhat more difficult. The following birational morphism is a simplification of an example due to Cassou-Nogues and Russell [2]:

$$
\begin{gathered}
x \rightarrow x^{4} y^{2}-2 x^{3} y+x^{2}+x y, \\
y \rightarrow x^{6} y^{3}-3 x^{5} y^{2}+3 x^{4} y+2 x^{3} y^{2}-x^{3}-3 x^{2} y+x+y .
\end{gathered}
$$

We explain this example in Section 2, after the proof of Theorem 1.

\section{PEAK REDUCTION}

The "peak reduction" method is a simple but rather powerful combinatorial technique with applications in many different areas of mathematics as well as theoretical computer science. It was introduced by Whitehead, who used it to solve an important algorithmic problem concerning automorphisms of a free group (see e.g. [6]). Since then, this method has been used to solve various problems in group theory, topology, combinatorics, and probably in some other areas as well.

In general, this method is used to find some kind of canonical form of a given object $P$ under the action of a given group (or a semigroup) $T$ of transformations. The idea behind the method is rather simple: one chooses the complexity of an object $P$ one way or another, and declares a canonical form of $P$ to be an object $P^{\prime}$ whose complexity is minimal among all objects $t(P), t \in T$. To actually find a canonical form, or a "canonical model", $P^{\prime}$ of a given object $P$, one tries to arrange a sequence of sufficiently simple transformations so that the complexity of an object decreases at every step. To prove that such an arrangement is possible, one uses "peak reduction"; that means, if in some sequence of simple transformations the complexity goes up (or remains unchanged) before eventually going down, then there must be a pair of consecutive simple transformations in the sequence (a "peak") such that one of them increases the complexity (or leaves it unchanged), and then the other one decreases it. Then one tries to prove that such a peak can always be reduced.

In the commutative algebra context, objects are polynomials; their complexity is their degree; the group of transformations is the group of polynomial automorphisms; simple transformations are elementary and linear automorphisms. (An elementary automorphism is one that changes just one variable.)

We have used this technique in our earlier paper 8 to contribute toward a classification of two-variable polynomials having classified, up to an automorphism, polynomials of the form $a x^{n}+b y^{m}+\sum_{i m+j n \leq m n} c_{i j} x^{i} y^{j}$ (i.e., polynomials whose 
Newton polygon is either a triangle or a line segment). Later, Wightwick [7] used the idea of peak reduction in combination with the splice diagrams technique due to Eisenbud and Neumann [5] to classify all two-variable polynomials over $\mathbf{C}$ up to an automorphism. More details and results can be found in our survey [9].

Here we use this technique to prove our statements.

Proof of Theorem 1.1 and Proposition 1.2. Consider the direct product $K[x, y] \times$ $K[x, y]$ of two copies of the polynomial algebra $K[x, y]$, and introduce the following elementary transformations (ET) that can be applied to elements of this direct product:

(ET1) $(u, v) \longrightarrow\left(\frac{u+a}{c \cdot v+b}, v\right)$ for arbitrary $a, b, c \in K$, with the denominator not 0 ; $\left(\mathbf{E T 1}^{\prime}\right)(u, v) \longrightarrow\left(u, \frac{v+a}{c \cdot u+b}\right)$ for arbitrary $a, b, c \in K$, with the denominator not 0 ; (ET2) $(u, v) \longrightarrow(u+q(v), v)$ for an arbitrary polynomial $q(v)$;

$\left(\mathbf{E T 2}^{\prime}\right)(u, v) \longrightarrow(u, v+q(u))$ for an arbitrary polynomial $q(u)$;

(ET3) $(u, v) \longrightarrow(v, u)$.

Transformations (ET1) or (ET1') are only applied if the corresponding ratio is a polynomial.

It is clear that a birational morphism $x \rightarrow u, y \rightarrow v$ is a product of polynomial automorphisms and mappings of the form $x \rightarrow x, y \rightarrow x \cdot y$ if and only if the pair $(u, v)$ can be taken to $(x, y)$ by a sequence of elementary transformations (ET1)-(ET3).

We are now going to use "peak reduction" to show that, if the sum of the degrees of a pair of polynomials can be reduced by a sequence of elementary transformations (ET1)-(ET3), then it can be reduced by a single elementary transformation.

Obviously, (ET3) cannot change the sum of the degrees; also, a single (ET1) or $\left(\mathrm{ET}^{\prime}\right)$ can only decrease the sum of the degrees, unless $c=0$. Thus, up to a symmetry, there are only the following possibilities for a "peak":

(1) (ET3) followed by one of the (ET1), (ET1'), (ET2), or (ET2');

(2) (ET2) followed by $\left(\mathrm{ET}^{\prime}\right)$;

(3) (ET2) followed by (ET1) or $\left(\mathrm{ET} 1^{\prime}\right)$.

The first possibility is trivial: for example, (ET3) followed by (ET1) is the same as $\left(\mathrm{ET}^{\prime}\right)$ followed by (ET3). Therefore, if the sum of the degrees could be reduced by (ET3) followed by (ET1), then it could also be reduced by (ET1') followed by (ET3), hence by just $\left(\mathrm{ET}^{\prime}\right)$.

The second possibility was handled in [8]; it turns out to be easy, too.

In (3), if (ET2) is followed by $\left(\mathrm{ET}^{\prime}\right)$, we get the pair $\left(u+q(v), \frac{v+a}{c \cdot[u+q(v)]+b}\right)$. Since we are under the assumption that (ET2) does not decrease the sum of the degrees, we have $\operatorname{deg}(u+q(v)) \geq \operatorname{deg}(u)$. Therefore, we have two possibilities:

(i) $\operatorname{deg}(u+q(v))>\operatorname{deg}(u)$. In this case, $\operatorname{deg}(u+q(v))=\operatorname{deg}(q(v))=\operatorname{deg}(q)$. $\operatorname{deg}(v)$, and we conclude that the polynomial $q$ must be linear; otherwise, $v+a$ cannot be divisible by $c \cdot[u+q(v)]+b$. Moreover, $q$ has to be a constant, since otherwise the ratio $\frac{v+a}{c \cdot[u+q(v)]+b}$ is a constant, but no pair of the form $(u, c), c \in K$, can be taken to $(x, y)$ by a sequence of (ET1)-(ET3). But if $q(v)$ is a constant, then $\left(\mathrm{ET}^{\prime}\right)$ cannot change the sum of the degrees.

(ii) $\operatorname{deg}(u+q(v))=\operatorname{deg}(u)$. In this case, $\operatorname{deg}(u) \geq \operatorname{deg}(q(v))$. So again, the polynomial $q$ must be linear; otherwise, $v+a$ cannot be divisible by $c \cdot[u+q(v)]+b$. As in (i) above, we conclude that $q$ has to be a constant, in which case (ET1') cannot change the sum of the degrees. 
Thus, we are left with the crucial possibility where (ET2) is followed by (ET1) with $c \neq 0$. The result of applying this pair of elementary transformations to $(u, v)$ is $\left(\frac{u+q(v)+a}{c \cdot v+b}, v\right)$. The polynomial $q(v)$ can be written as $r(c \cdot v+b)$ for some other polynomial $r$. Then

$$
\left(\frac{u+q(v)+a}{c \cdot v+b}, v\right)=\left(\frac{u+r(c \cdot v+b)+a}{c \cdot v+b}, v\right)=\left(\frac{u+r_{1}(c \cdot v+b)+a_{1}}{c \cdot v+b}, v\right),
$$

where the polynomial $r_{1}$ has zero constant term.

Since $r_{1}(c \cdot v+b)$ is divisible by $c \cdot v+b$, we get

$$
\left(\frac{u+r_{1}(c \cdot v+b)+a_{1}}{c \cdot v+b}, v\right)=\left(\frac{u+a_{1}}{c \cdot v+b}+r_{2}(c \cdot v+b), v\right) .
$$

This means, in particular, that $u+a_{1}$ is divisible by $c \cdot v+b$. But then a single (ET1) would reduce the sum of the degrees of the pair $(u, v)$. This completes the "peak reduction".

Now to get an algorithm claimed in the statement of Theorem 1, we first have to show that one can effectively determine whether a single elementary transformation can reduce the sum of the degrees of a given pair of polynomials. For (ET2) or $\left(\mathrm{ET} 2^{\prime}\right)$, this is well known (see e.g. [3, Theorem 6.8.5]). For (ET1) or $\left(\mathrm{ET} 1^{\prime}\right)$, the procedure is quite straightforward. Suppose we want to find out whether, say, $u(x, y)+a$ is divisible by $v(x, y)+b$ for some $a, b \in K$ (we can clearly assume that $c=1$ ). We apply the usual "long division" algorithm with respect to some fixed term ordering (see e.g. [1]). In the end, the divisibility condition translates into a system of polynomial equations over $K$ in a single variable $b$, complemented by an equation $a=p(b)$ for some polynomial $p$. The solvability of such a system over an algebraically closed field can be decided by using the Gröbner bases technique (see e.g. [1]). (Of course, an actual solution may not be found in general.)

If the system has no solutions, then a single elementary transformation cannot reduce the sum of the degrees of a given pair of polynomials, and we conclude that our birational morphism is not a product of simple affine contractions. If the system has a solution, then we apply the corresponding elementary transformation, keeping our parameters $a, b$, etc. as variables, because we do not have explicit values for them. Then, for the new pair of polynomials, we do the same thing: we check if a single elementary transformation can reduce the sum of the degrees. This will yield another system of polynomial equations over $K$ that expands the previous system by introducing new variables and new equations. Again, it can be decided whether or not this system has a solution.

This procedure will obviously terminate in a number of steps not exceeding the sum of the degrees of the original pair of polynomials. This completes the proof.

Example 1. The birational morphism

$$
\begin{gathered}
x \rightarrow u(x, y)=x^{4} y^{2}-2 x^{3} y+x^{2}+x y, \\
y \rightarrow v(x, y)=x^{6} y^{3}-3 x^{5} y^{2}+3 x^{4} y+2 x^{3} y^{2}-x^{3}-3 x^{2} y+x+y,
\end{gathered}
$$

mentioned in the Introduction, is constructed as follows. Start with the birational morphism $\left(x y+1, x^{2} y+x\right)$, apply $x \rightarrow x+y, y \rightarrow y$ to get $((x+y) y+1$, $\left.(x+y)^{2} y+(x+y)\right)$. Then apply $x \rightarrow \frac{1}{x}, y \rightarrow-x+x^{2} y$ (this is a bijective morphism of $K(x, y))$ to get the above example. 
A single transformation $\left(\mathrm{ET}^{\prime}\right)$ cannot reduce the sum of the degrees of the pair $(u, v)$ because the degree of $v$ is not divisible by the degree of $u$ (cf. [8]). Neither can a single $\left(\mathrm{ET}^{\prime}\right)$ reduce the sum of the degrees: a straightforward check shows that $v(x, y)+a$ is not divisible by $c \cdot u(x, y)+b$ for any $a, b, c \in K$. Therefore, by Proposition 1.2, this birational morphism is not a product of simple affine contractions.

Example 2. The birational morphism $x \rightarrow x, y \rightarrow y x^{2}+y$ is not a product of simple affine contractions over $\mathbf{R}$, by Proposition 1.2. However, over $\mathbf{C}$, a single $\left(\mathrm{ET}^{\prime}\right)$ can reduce the degree of $y x^{2}+y$ since $y x^{2}+y=y\left(x^{2}+1\right)=y(x+i)(x-i)$. It is now easy to see that over $\mathbf{C}$, this birational morphism is a product of simple affine contractions.

\section{ACKNOWLEDGMENT}

We are grateful to Andrew Campbell for helpful comments.

\section{REFERENCES}

[1] W. Adams and P. Loustaunau, An introduction to Gröbner bases, Graduate Studies in Mathematics, vol. 3, American Mathematical Society, Providence, RI, 1994. MR 95g:13025

[2] P. Cassou-Nogues and P. Russell, On some birational endomorphisms of the affine plane, preprint.

[3] P. M. Cohn, Free Rings and their Relations, second edition, London Mathematical Society Monographs, vol. 19, Academic Press, London, 1985. MR 87e:16006

[4] D. Daigle, Birational endomorphisms of the affine plane, J. Math. Kyoto Univ. 31 (1991), 329-358. MR 92k:14012

[5] D. Eisenbud and W. D. Neumann, Three-dimensional link theory and invariants of plane curve singularities, Ann. Math. Studies 110, Princeton University Press, Princeton, NJ, 1985. MR 87g:57007

[6] R. Lyndon and P. Schupp, Combinatorial Group Theory, reprint of the 1977 edition, in Classics in Mathematics, Springer-Verlag, Berlin, 2001. MR 2001i:20064

[7] P. Wightwick, Equivalence of polynomials under automorphisms of $\mathbb{C}^{2}$, J. Pure Appl. Algebra 157 (2001), 341-367. MR 2002c:14091

[8] V. Shpilrain and J.-T. Yu, Embeddings of curves in the plane, J. Algebra 217 (1999), 668-678. MR 2001f:13012

[9] V. Shpilrain and J.-T. Yu, Peak reduction technique in commutative algebra: a survey, in: Combinatorial and Computational Algebra (Hong Kong, 1999), 237-247, Contemp. Math. 264, Amer. Math. Soc., Providence, RI, 2000. MR 2001m:14087

Department of Mathematics, The City College of New York, New York, New York 10031

E-mail address: shpil@groups.sci.ccny.cuny.edu

$U R L:$ http://www.sci.ccny.cuny.edu/ ${ }^{\text {shpil/ }}$

Department of Mathematics, The University of Hong Kong, Pokfulam Road, Hong KONG

E-mail address: yujt@hkusua.hku.hk

$U R L:$ http://hkumath.hku.hk/ jtyu 